\title{
ASPECTOS HIDROGEOMORFOLOGICOS E A DISTRIBUIÇÃO ESPACIAL DA HANSENÍASE EM GUARAPUAVA - PR
}

\author{
HYDROGEOMORPHOLOGICAL ASPECTS AND SPATIAL DISTRIBUTION OF LEPROSY IN \\ GUARAPUAVA - PR
}

\author{
Edivaldo Geffer \\ Doutorando em Geografia pela Universidade Estadual do Centro-Oeste \\ Instituto Federal Mato Grosso do Sul \\ edivaldo.geffer@ifms.edu.br
}

Christian Junior Licheski Diaz Mestre em Geografia pela Universidade Estadual do Centro-Oeste Universidade Estadual do Centro-Oeste licheski.diaz@gmail.com

\begin{abstract}
RESUMO
A geografia da saúde busca contribuir com uma medicina preventiva, evidenciando os aspectos geográficos que contribuem para o desenvolvimento e incidência das doenças. Neste sentido foi feito um estudos sobre Hanseníase, analisando geograficamente a partir das concepções da geografia da saúde e de fatores Hidrogeomorfológicos na área urbana da cidade de Guarapuava-PR. Para tal, foram utilizados ferramentas do Sistema de Informação Geográfica (SIGs), para qualificação, distribuição, mapeamento e elaboração de gráficos a respeito do desenvolvimento dos focos destes agravos, os dados foram disponibilizados pelo Ambulatório Municipal de Pneumologia e Dermatologia Sanitária (AMPDS). Foi investigado a existência ou não de relação entre fatores hidrogeomorfológicos e a distribuição espacial de 304 casos confirmados de hanseníase residentes na área urbana da cidade de Guarapuava, entre 2001 a 2013. Trabalhos in loco foram desenvolvidos para analisar as possíveis fontes de contaminação, verificando pessoalmente os prováveis fatores que influenciam o aparecimento e o desenvolvimento destas doenças. Dentre os resultados obtidos destaca-se que as áreas com concentração de casos de Hanseníase em Guarapuava são regiões periféricas, carentes de infraestrutura básica de saneamento. Tendo a faixa etária da população mais atingida, a população economicamente ativa. Constatou-se ainda que muitas das residências com casos registrados observadas apresentam solo exposto e proximidade a corpos de água. A espacialização dos casos de Hanseníase torna-se uma ferramenta fundamental uma vez que, possibilita ações por parte do poder público em locais estratégicos do espaço urbano com maior concentração de casos.
\end{abstract}

Palavras Chave: Saúde. Geoprocessamento. Hanseníase.

\begin{abstract}
The geography of health seeks to contribute to preventive medicine, highlighting the geographical aspects that contribute to the development and incidence of diseases. In this sense, a study on Hansen's disease was made, analyzing geographically from the conceptions of health geography and Hydrogeomorphological factors in the urban area of the city of Guarapuava-PR. To this end, tools from the Geographic Information System (GIS) were used to qualify, distribute, map and graph the development of the outbreaks of these diseases, the data were made available by the Municipal Ambulatory of Pulmonology and Sanitary Dermatology (AMPDS). The existence or not of a relationship between hydrogeomorphological factors and the spatial distribution of 304 confirmed cases of leprosy living in the urban area of the city of Guarapuava, between 2001 and 2013, was investigated. On-site work was carried out to analyze the possible sources of contamination, checking personally the probable factors that influence the appearance and development of these diseases. Among the results obtained, it is noteworthy that the areas with a
\end{abstract}

Recebido em: 13/02/2020

Aceito para publicação em: 01/07/2020. 
concentration of leprosy cases in Guarapuava are peripheral regions, lacking basic sanitation infrastructure. Having the age group of the population most affected, the economically active population. It was also found that many of the homes with registered cases observed have exposed soil and proximity to bodies of water. Spatialization of leprosy cases becomes a fundamental tool since it allows actions by the public authorities in strategic locations in the urban space with a higher concentration of cases.

Keywords: Health. Geoprocessing. Hansen's disease.

\section{INTRODUÇÃO}

A Geografia da Saúde, um dos mais novos ramos da Geografia, em relação à formação milenar da ciência geográfica como um todo, possibilita a análise da incidência de distribuição espacial e temporal de doenças com fatores sociais, geográficos e biológicos. A integração destes fatores norteia a adoção de ações sociais para as melhorias nas condições de saúde e, consequentemente da qualidade de vida da sociedade (GESLER E KEARNS 2002).

Segundo Lacaz (1972, p.1) a Geografia Médica, Patologia Geográfica ou Geopatologia pode ser entendida como a "disciplina que estuda a geografia das doenças, isto é, a patologia à luz dos conhecimentos geográficos". Barcellos (2008), cita que a geografia médica se classificava em dois fatores essenciais: os fatores patológicos, relacionados aos agentes causadores, vetores, hospedeiros, reservatórios e o homem, assim como também os fatores geográficos, seja físico, humanos, sociais e biológicos. Tal fato levou a uma mudança em seu "título" para "Geografia da Saúde".

No entanto, sua concepção epistemológica ainda está em consolidação, as discussões e os estudos desenvolvidos estão "mais direcionados, planejados e com o objetivo de desenvolver ações de prevenção, ou seja, propor trabalhos dentro da perspectiva da medicina preventiva" (PEREHOUSKEI e BENADUCE, 2007 p. 35).

Para Mohanty (2016), as condições ambientais, físicas e sociais, na qual, o indivíduo e/ou uma sociedade está inserida, condicionam a ocorrência, a distribuição, o tratamento e a manutenção de doenças. A diversidade, a complexidade e a dimensão continental do Brasil, em razão das diferenças regionais, tornam ainda mais importantes os estudos investigativos das condições ambientais condicionante de doenças.

No Brasil, as doenças que podem colocar em risco a saúde das coletividades devem ser notificadas no Sistema de informação de Agravos de Notificação (SINAN), quando identificadas pelos agentes de saúde. Dentre as doenças, a Hanseníase (HAN), está descrita na Lista de Doenças de Notificação Compulsória (LDNC) (BRASIL, 2017), baseada na Portaria $N^{\circ} 4$ de 28 de setembro de 2017, que define a LDNC, agravos e eventos de saúde pública e privados em todo o território nacional. Esta lista foi desenvolvida pelo SDNC.

Este sistema prevê o potencial das doenças, como: magnitude, potencial de disseminação, transcendência, vulnerabilidade, disponibilidade de medidas de controle, compromissos internacionais com programas de erradicação, entre outros fatores. A hanseníase é uma doença de notificação compulsória semanal (NCS), apesar de não ser doença endêmicas apenas do Brasil, estando presente em várias regiões do planeta.

De acordo com os dados da Secretaria Estadual de Saúde, Guarapuava é o quinto município do Estado do Paraná com maior número de incidência de hanseníase, (PARANÁ, 2019). O coeficiente de detecção em Guarapuava de HAN é de 14,0 casos a cada 100.000 mil habitantes, enquanto que a média nacional é de 15,9 da HAN, a cada 100.000 mil habitantes (BRASIL, 2017).

Apesar de Guarapuava apresentar um coeficiente de detecção menor do que a média nacional de hanseníase, segundo Brasil (2017), o índice de detecção da hanseníase é considerado elevado se comparado às médias do estado do Paraná, a qual é 10,2 casos a cada 100.000 mil habitantes.

A adoção de estratégias para erradicação da hanseníase exige conhecimento da doença, de suas causas, agentes transmissores, visando sua redução e/ou eliminação. As formas clínicas da hanseníase são: Hanseníase Indeterminada $(\mathrm{HI})$, Hanseníase Tuberculóide (HT), Hanseníase Dimorfa (HD) e Hanseníase Virchowiana (HV) (BRASIL, 2017). 
A transmissão do bacilo Mycobacterium leprae é muito maior que a taxa de ataque da doença propriamente dita. Apenas $5 \%$ dos indivíduos infectados demostram os sintomas clínicos da doença, em razão de estarem com sistema imunológico indefeso (WORLD HEALTH ORGANIZATION, 1988).

Kazda (1981) realizou estudos em regiões endêmicas da doença hanseníase, na Noruega, Costa do Marfim, Portugal, Peru, Índia, e no estado de Louisiana nos EUA. A pesquisa analisou o solo, água e a vegetação, também realizou inoculação do bacilo da hanseníase em patas de animais, tatus e camundongos, e por meio de testes bioquímicos avaliaram a contaminação ou não do solo, pelo bacilo de Hansen. A pesquisa revelou o crescimento característico da hanseníase nas patas dos camundongos e dos tatus gerando resultado positivo em ambos os testes bioquímicos. Kazda (1986) realizou uma nova experiência em Bombain na Índia, o qual identificou a presença da Mycobacterium leprae no solo.

Na cidade de Guarapuava, dados do Ambulatório Médico de Pneumologia e Dermatologia Sanitária (AMPDS), Prefeitura Municipal e Secretaria de Saúde, fonte básica de dados para esta pesquisa, indicam a existência de focos de casos de hanseníase, que ocasionam perda de mão-de-obra ativa e geram gastos e demanda ao sistema de saúde.

Desta forma, nota-se a necessidade da redução do número de casos de hanseníase. Para tal é fundamental conhecer os fatores condicionantes da ocorrência de hanseníase em Guarapuava, sejam eles físicos ou sociais. Conforme o Brasil (2011), estudos comprovam existir relação entre a incidência das doenças a fatores sociais como falta de saneamento básico, acesso à saúde, condições de trabalho, moradia, alimentação e lazer.

Contudo, poucos são os estudos que avaliam a relação existente entre casos de hanseníase aos aspectos físicos, como temperatura e umidade (BRASIL, 2014), e são raros estudos sobre a relação entre os aspectos hidrogeomorfológicos e a incidência da doença.

Com base nisso a pesquisa busca contextualizar a ocorrência de determinados agravos à saúde e fatores socioambientais de risco. Essa observação sobre o meio ambiente deve estar comprometida com a totalidade salientando aspectos físicos da área, como topografia, condições e densidade das habitações, sistema de drenagem, eventual presença de esgoto a céu aberto, acúmulo de lixo, córregos, e outros aspectos visíveis que indiquem diferenças nas condições de vida entre os residentes da área (PEREHOUSKEI e BENADUCE, 2007, p.42).

A compreensão sobre a relação do endemismo da hanseníase, aspectos hidrogeomorfológicos e desigualdades socioambientais específicas pode subsidiar ações de prevenção e mitigação dessas doenças na cidade de Guarapuava-PR. Em campo foram analisados as condições das residências, material que foi construída, a direção da face da residência, saneamento básico, vegetação, lixo, entulho, animais, corpos hídricos, tipos de vertentes, temperatura, umidade, insolação e vento.

\section{MATERIAIS E MÉTODOS}

O estudo investigou a existência ou não de relação entre fatores hidrogeomorfológicos e a distribuição espacial de 304 casos confirmados de hanseníase residentes na área urbana da cidade de Guarapuava-PR, entre 2001 a 2013, registrados no Ambulatório Municipal de Pneumologia e Dermatologia Sanitária (AMPDS), vinculado à Secretaria Municipal de Saúde de Guarapuava-PR, sendo espacializados e avaliados pela sobreposição aos planos de informações hidrogeomorfológicos (hipsometria, declividade e proximidade de corpos de água) e observações de campo.

Utilizando-se do software ArcGis 10.2 e de bases cartográficas, das cartas topográficas de Guarapuava OSG 2838/3 SG.22-V-D-III-3 e 2837/4 SG.22-V-D-II-4, na escala de 1:50.000, de 1991, editadas pelo Serviço Geográfico do Exército Brasileiro; e de arquivos vetorizados representando o perímetro urbano oficial, os rios principais, as curvas de nível (equidistância entre as curvas de nível de $5 \mathrm{~m}$ ) e os pontos cotados, na escala de 1:12.000, cedida pelo Centro de Estudos e Planejamento Urbano de Guarapuava da Prefeitura Municipal de Guarapuava - CEPLUG/PMG obteve-se o modelo digital do terreno, a hipsometria e a declividade.

Os dados de casos novos confirmados de hanseníase entre 2001 a 2013 obtidos junto ao Ambulatório Municipal de Pneumologia e Dermatologia Sanitária (AMPDS), vinculada à Secretaria Municipal de Saúde de Guarapuava foram espacializados. Os dados de hanseníase foram ano de notificação, a data; endereço com nome da rua e o número da residência, bairro, sexo, idade do 
paciente, forma clínica, o modo de entrada e o exame baciloscópico.

Os dados de hanseníase foram inicialmente tabulados em planilhas do Excel do Software Microsoft Office 2010, por caso registrado, contendo nas linhas os casos e nas colunas as informações citadas acima, respectivamente conforme a doença. Em um segundo momento, selecionaram-se as ocorrências registradas no perímetro urbano da cidade de Guarapuava, recorte espacial do presente estudo, de forma que tais informações foram geocodificadas para poder realizar a distribuição espacial em mapas.

Posteriormente, foram espacializados sobre a área urbana de Guarapuava, utilizando-se do endereço por geocodificação. Na espacialização os casos foram divididos em planos de informações anuais, ou seja, cada layer continha dados dos casos registrados no ano e doenças. Para cada registro de doença era inserido um ponto no mapa da sua localização. A localização do ponto deu-se por meio do endereço.

A geocodificação consistiu no processo de conversão de endereços em coordenadas geográficas, que podem ser usadas para inserir marcadores ou posicionar pontos no mapa. Os casos/pontos de ocorrência da hanseníase foram localizados com o auxílio do Software Google Earth Pro e salvos em formato KML. Os dados, posteriormente, foram importados para o ArcGis 10.2 e QGIS.

$\mathrm{Na}$ análise da distribuição da hanseníase frente aspectos hidrogeomorfológicos utilizou-se da sobreposição dos casos (pontos) e não dos mapas de densidade das doenças de Kernel obtidos no software QGIS (mapa de calor ou heatmap). Uma vez, que a densidade é calculada com base no número de pontos de um local, ou seja, quanto maior for o número de pontos agrupados (cluster), a percepção da densidade será maior.

Em campo avaliou-se aspectos hidrogeomorfologicos e socioambientais, como qualidade ambiental local, tipo de habitação, face de exposição da residência, umidade, presença de corpos hídricos e vegetação. Tendo como finalidade estabelecer possíveis correlações dos casos das doenças pesquisadas com o local onde as pessoas residem.

\section{CARACTERIZAÇÃO DA ÁREA DE ESTUDO}

Segundo o IBGE (2019), o município de Guarapuava possuía uma população estimada de 175.799 mil habitantes, em 2016. Localizado na porção Centro Sul do Estado do Paraná, com área territorial de $3.117,01 \mathrm{~km}^{2}$ e densidade demográfica de 55,38 habitantes por $\mathrm{km}^{2}$ (IPARDES, 2015).

O município de Guarapuava faz divisa com os seguintes municípios: ao norte, Campina do Simão e Turvo, ao sul com Pinhão, à leste com Prudentópolis e Inácio Martins e a oeste com Candói, Cantagalo e Goioxim (Figura 1).

Para Maack (2002), Guarapuava caracteriza-se por uma paisagem de campo limpo, capões e matas de galeria associadas às araucárias. O bioma predominante é Mata Atlântica. A Floresta com Araucária é uma formação fitogeográfica especial do Bioma Mata Atlântica, cujo desenvolvimento se relaciona à altitude acima dos $500 \mathrm{~m}$, ocupando uma zona caracteristicamente subtropical em áreas elevadas do Planalto Meridional Brasileiro.

Thomaz e Vestena (2003, p. 29), definiram o clima de Guarapuava, a partir de uma série histórica de dados "como subtropical mesotérmico úmido, caraterizado pela ausência da estação seca, com verões frescos e invernos moderados."

De modo geral, as chuvas são bem distribuídas ao longo do ano, com precipitações médias mensais acima de $100 \mathrm{~mm}$ e anuais entre 1.700 a $2.300 \mathrm{~mm}$. Os meses de outubro e janeiro são os mais chuvosos e os meses de agosto e julho os menos chuvosos. A temperatura média anual varia entre $16^{\circ} \mathrm{C}$ a $17^{\circ} \mathrm{C}$ (THOMAZ e VESTENA (2003).

A litologia no município de Guarapuava está enquadrada no Grupo São Bento, mais especificamente nas Formações Botucatu e Serra Geral. Na parte leste do município tem-se afloramento do Arenito Botucatu, na Escarpa da Esperança. O magmatismo fissural da Formação Serra Geral, gerou diferentes tipos de rochas, embora as rochas de natureza básica (principalmente basaltos) predominem em mais de $97,5 \%$ desta formação, há a ocorrência de rochas de natureza ácida (BINDA e BERTOTTI, 2008). 
Figura 1 - Localização do município de Guarapuava, no Estado do Paraná, Brasil

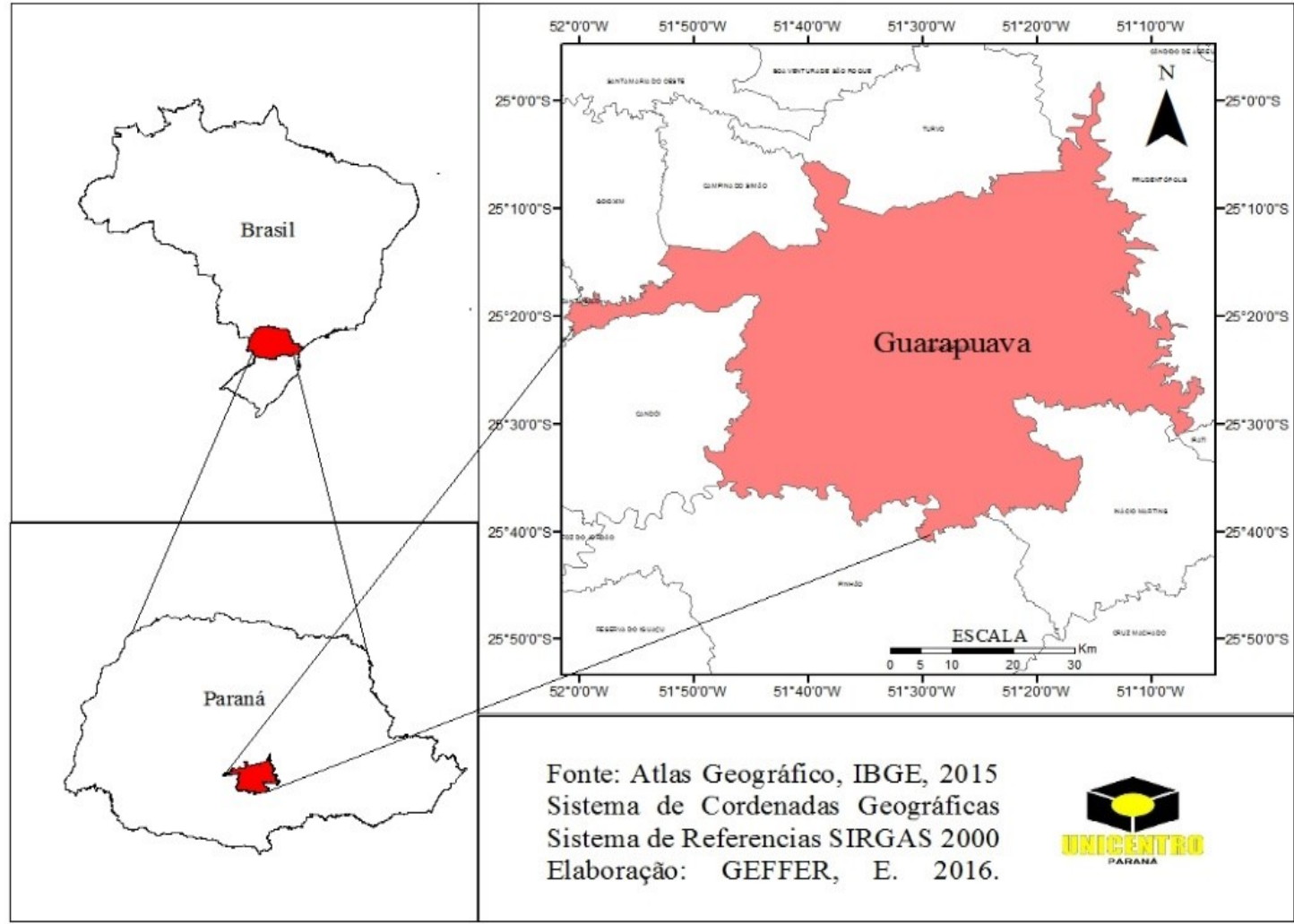

A hidrografia na região da cidade de Guarapuava é caracterizada por rios intermitentes e perenes, a densidade de drenagem é de $54 \mathrm{~km} / \mathrm{km}^{2}$ (OLIVEIRA et al, 2009). A bacia hidrográfica do rio Cascavel drena $81,82 \%$ da área urbana de Guarapuava (BINDA e BERTOTTI, 2008) no sentido nordestesudoeste, no entanto, seus afluentes Arroio Carro Quebrado, Arroio Barro Preto e Arroio do Engenho drenam o perímetro urbano no sentido Leste-Oeste, fato que demonstra um padrão de drenagem controlado pelo padrão estrutural do substrato rochoso (MINEROPAR, 1992).O rio Cascavel é afluente da margem direita do rio Jordão, que deságua no rio Iguaçu componente da bacia do rio Paraná.

Em relação ao processo de urbanização do município de Guarapuava, esse não foi diferente do contexto nacional, todavia deu-se tardiamente, mais especificadamente, a partir da década de 1970 . Os fatores econômicos e políticos foram os principais impulsionadores do desenvolvimento do município e do crescimento urbano em Guarapuava, uma vez que desde a sua formação em 1819, até 1970 , a sua população estava concentrada na área rural, cenário que foi drasticamente alterado a partir da década de 1980, período seguinte a população urbana ultrapassa a rural (SILVA, 1997).

Para SILVA (1997), esse processo de urbanização das cidades brasileiras, em especial o caso de Guarapuava, associado à ausência de planejamento, e a proliferação de ocupações irregulares, são responsáveis por uma gama de problemas socioambientais.

Em Guarapuava a intensidade da urbanização tornou as relações sociais e econômicas mais complexas, proporcionando uma grande demanda por espaços de moradia, e ocasionou uma série de problemas socioambientais, como a poluição do solo e da água e a "marginalização de parte da população" (VESTENA; SCHMIDT, 2009, p.69).

\section{RESULTADOS E DISCUSSÕES}

Destaca-se que a hanseníase é uma doença que pode estar relacionada aos microclimas, que influenciam no prolongamento da vida útil dos agentes etiológicos, principalmente ambientes úmidos e aquecidos, assim como também a relação da falta de saneamento básico e focos da doença (MOHANTY, 2016; Leano et al., 2019). No trabalho de campo ao visitar áreas mapeadas pelos casos, 
encontram-se muitas residências que estão em contato direto ou muito próximo com canais fluviais e áreas com solo sem cobertura. (Figura 2).

Figura 2 - Solo exposto e corpo de água próximo a residências de infectados.
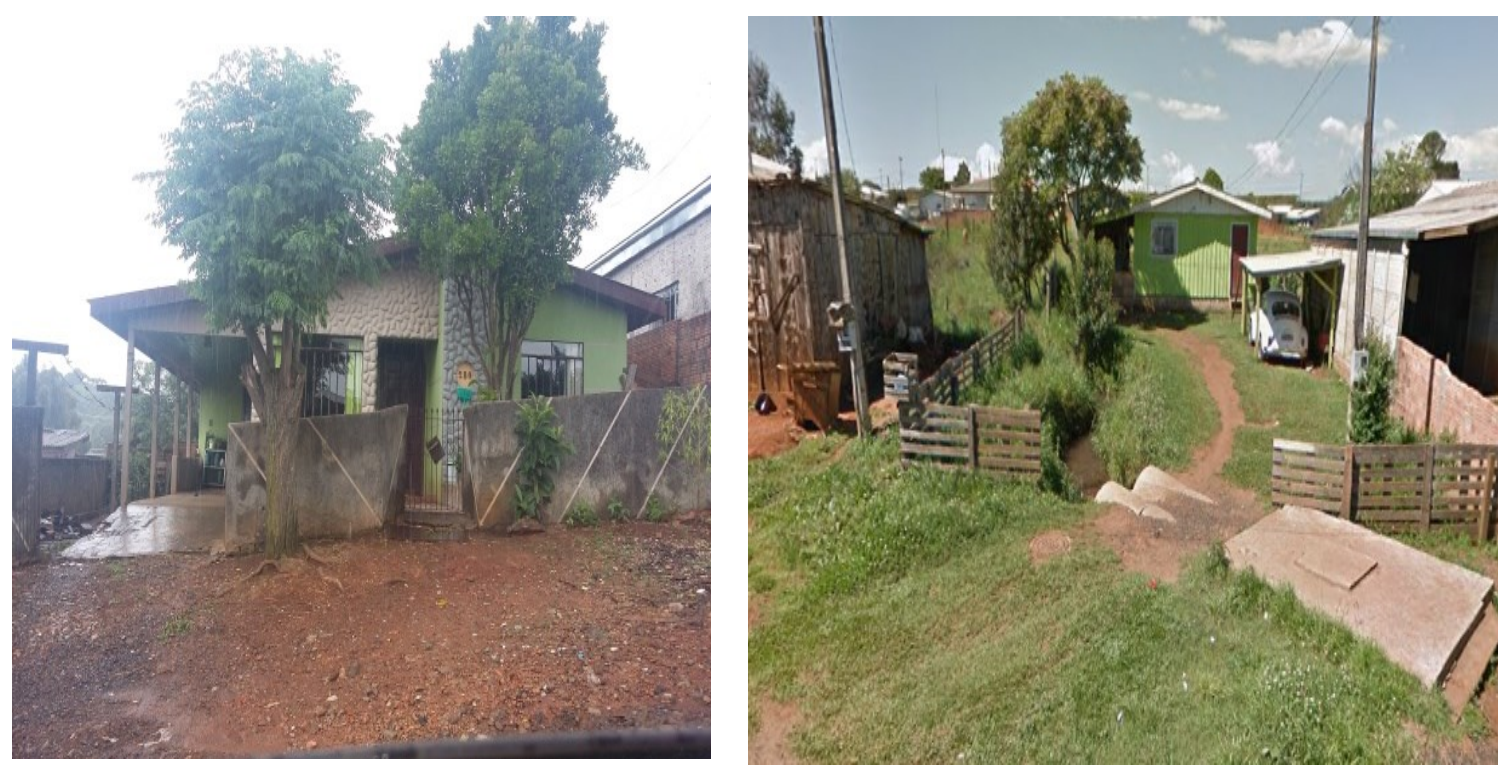

Fonte: Autores

Com base nos trabalhos de campo, constatou-se que muitas das residências de casos registrados observadas apresentam solo exposto e proximidade a corpos de água. Assim como muitos desses casos serem reincidentes, ou seja, a pessoa contraiu a doença mais de uma vez. Neste sentido este estudo corrobora com os resultados já obtidos de (KAZDA 1981, e MOHANTY 2016), uma vez que comprova que o solo e água podem se constituir como reservatórios ambientais da hanseníase.

Mohanty (2016) ao analisar amostras do solo e da água coletados em áreas com pacientes residentes, $4 \%$ das amostras de solo e $24,2 \%$ das de água possuíam o Mycobacterium leprae, sendo que em $21,8 \%$ dos locais continham o Mycobacterium leprae em ambas as amostras, em 37 amostras coletadas. Nas amostras de solo e água, na pesquisa desenvolvida por Mohanty (2016); contaminadas pelo bacilo constataram-se casos do tipo multibacilar e paucibacilar, indicando que o solo e a água podem conter o bacilo da Hanseníase, mesmo em áreas e locais distantes das residências das pessoas doentes.

Entre 2001 a 2013, conforme dados fornecidos pelo Ambulatório Municipal de Pneumologia e Dermatologia Sanitária (AMPDS), órgão vinculado à Secretaria de Saúde do Município de Guarapuava, PR, foram registrados 331 casos novos residentes de hanseníase, sendo 304 casos confirmados residentes na área urbana do município (Figura 3).

Os três bairros mais atingidos são bairros periféricos como o Boqueirão com 17,43\%, Morro Alto com $11,51 \%$ e industrial com $10,20 \%$ dos casos registrados. Se analisarmos o Centro da cidade poderá ser observado que o número de casos é bem inferior, cerca de $3,95 \%$ dos casos em relação aos três bairros citados.

Esses resultados corroboram com as pesquisas de (DIFFEY et al., 2000; MURTO et al., 2017). Que apontaram relação direta de aumento de ocorrências dos casos de Hanseníase em locais onde as pessoas possuem de modo geral baixa renda, nível de escolaridade baixo, moradias precárias falta de saneamento básico bem como um adensamento populacional elevado em bairros periféricos.

A falta de saneamento básico, as condições precárias de moradia no que diz respeito a infraestrutura das casas, as residências em locais que não poderiam ser ocupados devido a perigo eminente de inundações e/ou alagamentos, podem contribuem para o aumento de casos de hanseníase nesses bairros de Guarapuava. 
Figura 3 - Mapa da área de ocorrência dos casos de Hanseníase em Guarapuava/PR

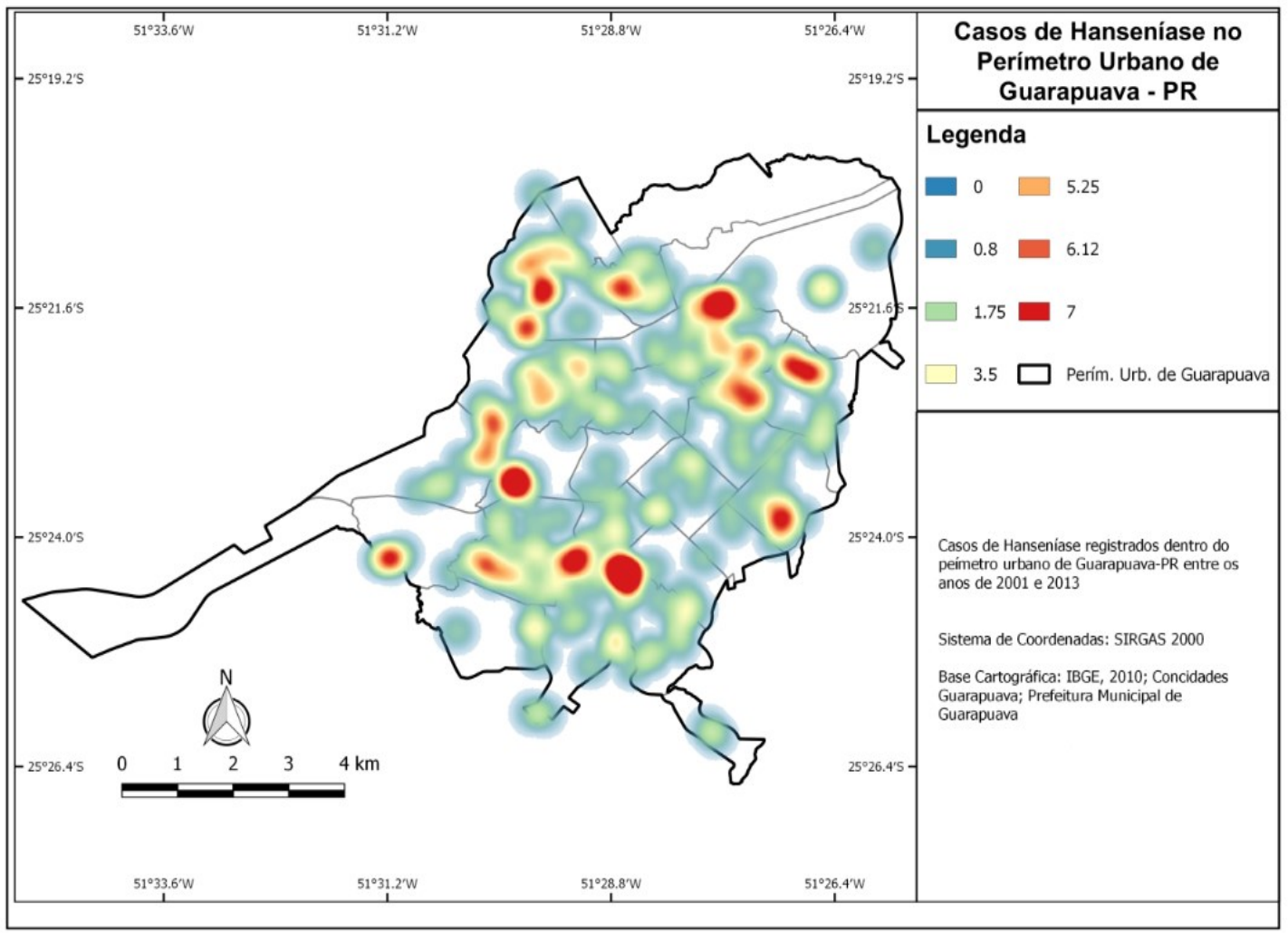

Elaboração: Autores

Conjuntamente com o fato que segundo OLIVEIRA et al (2009) a cidade de Guarapuava apresenta áreas propicias para o acúmulo de água, ficando os solos saturados em determinadas épocas do ano, o que condicionam a existência de banhados e charcos, habitat que podem propiciar microclima para o desenvolvimento dos agentes etiológicos de doenças. Na tabela 1 são apresentados os casos de hanseníase por bairro na cidade de Guarapuava.

Tabela 1 - Casos urbanos de HAN divididos por bairro em Guarapuava-PR.

\begin{tabular}{ccc}
\hline Bairro & Casos & $\%$ \\
\hline Boqueirão & 53 & 17,43 \\
Morro Alto & 35 & 11,51 \\
Industrial & 31 & 10,20 \\
Vila Bela & 26 & 8,55 \\
Jardim das Américas & 25 & 8,22 \\
São Cristovão & 19 & 6,25 \\
Bonsucesso & 18 & 5,92 \\
Vila Carli & 17 & 5,59 \\
Primavera & 14 & 4,61 \\
Santana & 12 & 3,95 \\
Centro & 12 & 3,95 \\
Santa Cruz & 10 & 3,29 \\
Conradinho & 10 & 3,29 \\
Batel & 8 & 2,63 \\
Alto Cascavel & 8 & 2,63 \\
Jordão & 4 & 1,32 \\
Alto da XV & 1 & 0,33 \\
Trianon & 1 & 0,33 \\
\hline
\end{tabular}

onte: Autores 
O sexo mais atingido pela hanseníase foi o sexo masculino com $58,55 \%$ do total dos casos, o sexo feminino registrou $41,45 \%$ dos casos. Em relação à faixa etária das pessoas, a idade mais atingida foi a economicamente ativa, registrando dos 18 aos 60 anos 68,75\% dos casos, seguido pelos idosos acima de 60 anos com 25,99\%, crianças de 0 a 12 anos apenas 2,63\% e adolescentes de 13 a 17 anos também com apenas $2,63 \%$ dos casos.

Sobre a faixa etária das pessoas infectadas pela hanseníase Araújo et al (2015) evidenciam em sua pesquisa, que indivíduos na fase da vida adulta e idosa bem como do sexo masculino de baixa renda, morando em moradias precárias com ausência de saneamento básico adequado são mais suscetíveis a hanseníase. Ainda segundo esse estudo o analfabetismo e ou poucos anos de estudo são fatores de risco para o adoecimento e sua posterior evolução para incapacidades físicas, uma vez que pode dificultar o reconhecimento das manifestações clinicas da doença, o acesso ao sistema de saúde e a compreensão das orientações de educação em saúde.

Verificou-se também neste trabalho se os fatores hidrogeomorfológicos estão ou não influenciando o desenvolvimento da hanseníase na cidade de Guarapuava. A figura 4 demonstra o modelo digital de elevação do terreno e a incidência dos casos de hanseníase na área urbana do município de Guarapuava/PR.

Figura 4 - Modelo digital do terreno e a incidência dos casos de hanseníase na área urbana de Guarapuava/PR

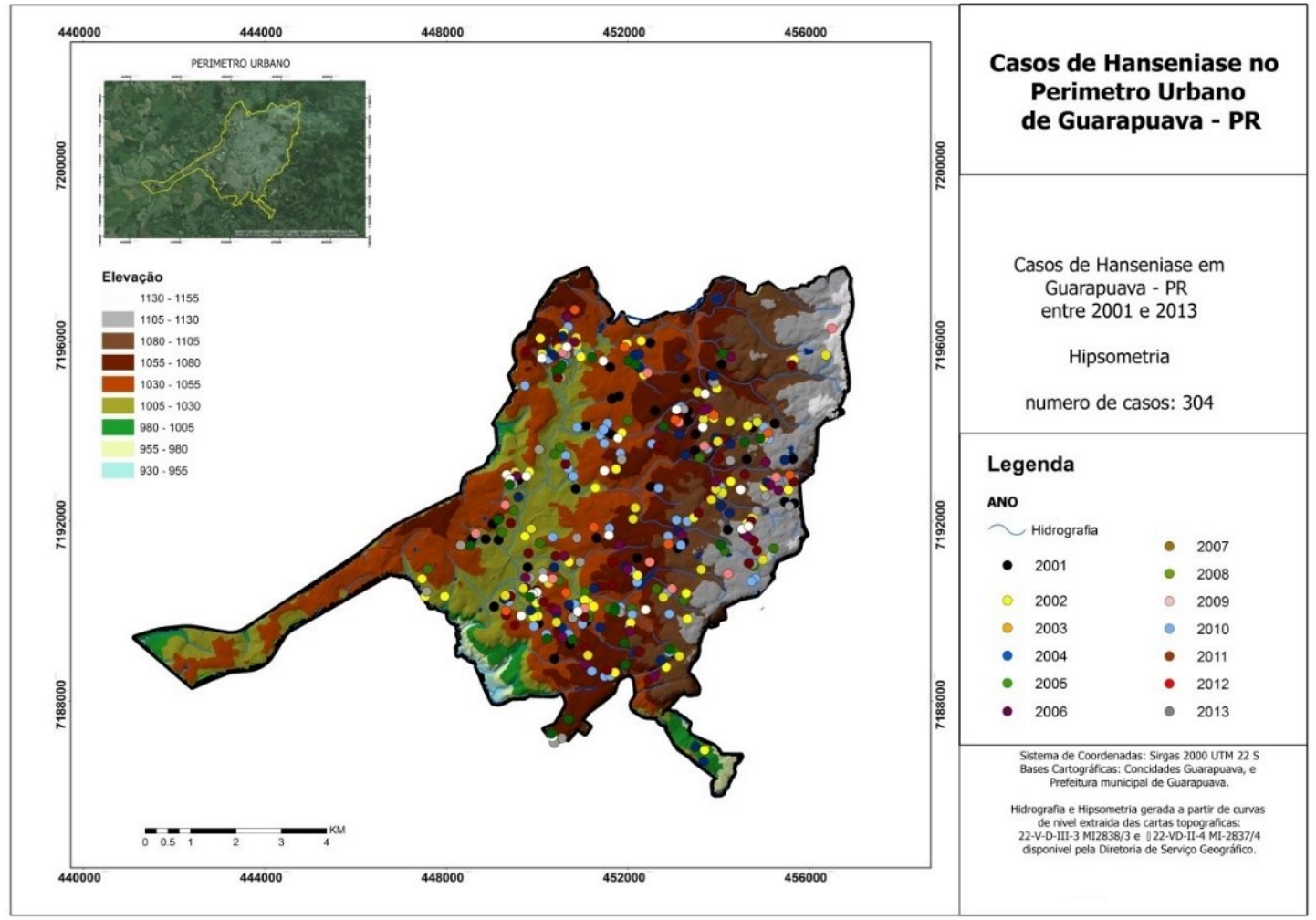

Elaboração: Autores.

Em relação a tais fatores hidrogeomorfológicos, destaca-se que 53,55\% dos casos de hanseníase residem a uma distância inferior a 100 metros de um corpo hídrico, sendo que 10,71\% dos casos de hanseníase estão a menos de 25 metros de distância. Este resultado constitui-se de fundamental importância mostrando que o contágio da hanseníase em alguns casos pode ter relação direta com a proximidade de residências a corpos hídricos. Uma vez que a água pode servir como agente de contágio contínuo da hanseníase como já demostrado em pesquisa realizada na Índia por (MOHANTY 2016). Nesta pesquisa ainda se apresentou evidencias de que mesmo em condições dos pacientes apresentarem melhoras em seu quadro clínico, o bacilo HAN continua presente, podendo contaminar o solo e a água e passar a contaminar outras pessoas. 
De modo geral, não se observou um padrão espacial na distribuição das doenças em função de níveis altimétricos, os casos ocorreram em diferentes altitudes. Verificando-se uma maior incidência de casos de hanseníase em altitude de 1030 a 1055 metros.

Contudo, uma relação direta entre as classes hipsométricas e a hanseníase não ficou evidente na cidade de Guarapuava. Ressalta-se que níveis de grandes variações altimétricas podem determinar a presença de microclimas, com condições de umidade e temperatura específicos.

Em relação a vegetação, com base nos dados e posterior trabalhos de campo para verificação, notou-se uma possível relação com a manutenção dos agentes da hanseníase, pois $60,71 \%$ dos casos de hanseníase possuíam vegetação próxima as suas residências, deixando alguns traços de que a vegetação pode ser, assim como o solo apontado por (KAZDA 1981, e MOHANTY 2016), um mantenedor e um propiciador de microclimas e reservatórios ambientais da hanseníase.

$\mathrm{Na}$ figura 05 tem-se a distribuição da HAN sobreposta a classes de declividade. $\mathrm{O}$ tipo de relevo obteve-se a partir das classes de declividade, segundo a EMBRAPA (1979). De modo geral, não se verificou a concentração dos casos de HAN em uma classe de declividade, ou tipo de relevo. Elas ocorrem com mais frequência em relevo suave ondulado com 3 a $8 \%$ de declividade. No entanto, observa-se a presença de casos em todos os tipos de relevo, e uma concentração de casos nos fundos de vales e encostas próximas e em certas regiões específicas da cidade.

Figura 5 - Classes de declividade e incidência dos casos de HAN na área urbana de Guarapuava/PR

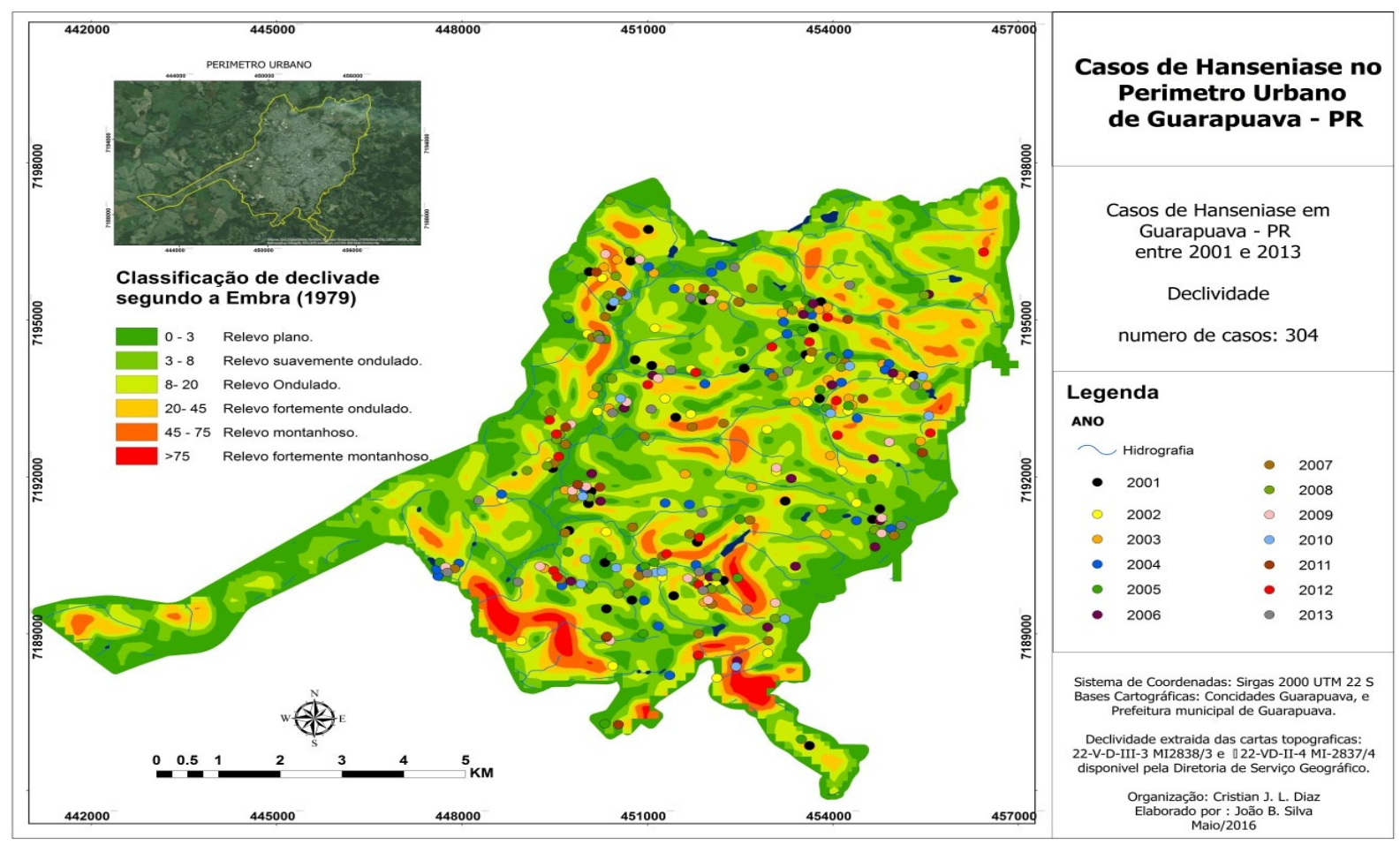

Elaboração: Autores

A pesquisa apontou que $71,03 \%$ dos casos estão registrados em uma declividade considerada suavemente ondulado a ondulado, poucos são os casos registrados em lugares montanhosos com uma inclinação superior a $45 \%$, ou mesmos em áreas planas onde apenas registrou-se $8,16 \%$ dos casos totais. 
Tabela 2 - Distribuição espacial dos casos de hanseníase em relação à declividade em GuarapuavaPR (Número de casos totais 304).

\section{CLASSE DE DECLIVIDADE (\%)}

NÚMERO DE CASOS DE HAN

$\%$

\begin{tabular}{lcc}
\hline+75 (Fortemente Montanhoso) & 4 & 1,31 \\
$45-75$ (Montanhoso) & 13 & 4,27 \\
$20-45$ (Fortemente ondulado) & 48 & 15,78 \\
$08-20$ (Ondulado) & 102 & 33,55 \\
$03-8$ (Suave ondulado) & 112 & 36,84 \\
$00-3$ (Plano) & 25 & 8,22 \\
\hline
\end{tabular}

Fonte: Autores

Constitui-se como uma ferramenta importante entender os fatores naturais e hidrogeomorfológicos em relação às doenças, uma vez que, doenças como a hanseníase, em muitos casos pode ter seu contágio associado ao relevo, vegetação, solo a presença de água, e a umidade do ambiente. Todavia, este estudo demonstra que os aspectos hidrogeomorfológicos não condicionam sua presença, ela é resultado da interação de todos os fatores no sistema ambiental.

Neste contexto, estudar os fatores "naturais" integrados aos antrópicos é fundamental. A exemplo segundo a companhia paranaense de águas e esgoto (SANEPAR), o município de Guarapuava apresenta um total de 50.576 domicílios, nos quais o saneamento básico representa apenas $86,54 \%$ de rede de esgoto, 99,06\% recebem água tratada, 93,03\% recebem coleta de lixo e 99,11\% possui acesso à energia elétrica.

Apesar de considerado elevado estes índices em relação à média nacional, ainda assim são 6.807 residências sem acesso a rede de esgoto, 474 famílias sem acesso a água tratada, 3.524 residências sem coleta de resíduos sólidos e 450 residências sem acesso à energia elétrica. Devido à falta destes serviços básicos, a saúde de muitas pessoas pode ser comprometida estando essas mais vulnerável a contrair inúmeros tipos de doenças, inclusive a hanseníase, como apontado neste estudo e nas pesquisas de (KAZDA 1981, e MOHANTY 2016).

A situação ainda piora quando as pessoas acumulam lixo na residência, a pesquisa detectou a presença resíduos sólidos descartados indevidamente no quintal das residências, de $25 \%$ dos casos de hanseníase

\section{CONCLUSÕES}

As distribuições espaciais da hanseníase, não apresentaram relação a fatores relacionados às formas de relevo, hipsométria e declividade na cidade de Guarapuava. As residências de infectados por hanseníase, em sua maioria encontram-se próximas a corpos de água.

A espacialização dos casos identificou regiões de concentração da doença na área urbana de Guarapuava. A hanseníase apresenta concentração de casos nos bairros Boqueirão, Morro Alto e Industrial. As áreas com concentração de casos de hanseníase em Guarapuava são regiões periféricas, carentes de infraestrutura básica de saneamento.

$\mathrm{Na}$ área urbana de Guarapuava existe um elevado índice de disseminação da hanseníase. A faixa etária da população mais atingida, pela hanseníase é a faixa economicamente ativa. A espacialização dos casos de hanseníase possibilita ações por parte do poder público em locais estratégicos do espaço urbano com maior concentração de casos. O conhecimento dos fatores condicionantes das doenças em nível local subsidia ações e medidas mitigadoras mais efetivas no combate as doenças.

\section{REFERÊNCIAS}

ARAÚJO, F. C. B. SOUZA, C. N.P. RAMOS, E. M. L. S. BRAGA, R. M. Aspectos associados à recidiva da hanseníase. Rev Bras Biom 2015. Disponível em: <http://jaguar.fcav.unesp.br/RME/fasciculos/v33/v33 n1/A4 Flavia Cristiane.pdf> Acesso em: 30 jun. 2020. 
BARCELLOS, C. A Geografia e o Contexto dos Problemas de Saúde. Rio de Janeiro: ABRASCO: ICICT: EPSJV, 2008.

BINDA, A. L; BERTOTTI, L. G. Mapeamento de Características Físicas do Relevo da Cidade de Guarapuava- PR Utilizando Técnicas de Geoprocessamento. Raega - O Espaço Geográfico em Análise, [S.I.], v. 16, out. 2008. ISSN 2177-2738. Disponível em: $<$ http://ojs.c3sl.ufpr.br/ojs/index.php/raega/article/view/11141>. Acesso em: 10 mar. 2019. https://doi.org/10.5380/raega.v16i0.11141

BRASIL, Ministério da Saúde. Manual de Recomendações para o Controle da TB no Brasil. Secretaria de Vigilância em Saúde; Departamento de Vigilância Epidemiológica. Brasília, DF, 2011. Disponível em: <http://www.cve.saude.sp.gov.br/htm/TB/mat tec/manuais/MS11 Manual Recom.pdf>

Acesso em:10 abril. 2019.

BRASIL, Ministério da Saúde. Diretrizes para vigilância, atenção e eliminação da hanseníase como problema de saúde pública. Secretaria de Vigilância em Saúde; Departamento de Vigilância Epidemiológica. $\quad$ Brasília, $\quad$ DF, $2016 . \quad$ Disponível em:< http://portalarquivos2.saude.gov.br/images/pdf/2016/fevereiro/04/diretrizes-eliminacao-hanseniase4fev16-web.pdf> Acesso 13 de maio de 2020.

DIFFEY, B; VAZ, M; SOARES, M. J; JACOB, A. J; PIERS L. S. The effect of leprosy-induced deformity on the nutritional status of index cases and their household members in rural South India: a socio-economic perspective. Eur J Clin Nutr. 2000. Disponível em: <https://www.ncbi.nlm.nih.gov/pubmed/10951513> 30 jun. 2020.

GIATTI, L. Fundamentos de Saúde Ambiental. Manaus: Editora da Universidade Federal do Amazonas, 2009.

GESLER, W. M. KEARNS, R. A. Culture/Place and Health. London and New York: 2002; p,182.

IPARDES. Caderno Estatístico Município de Guarapuava, mar. 2016. Disponível em: <http://www.ipardes.gov.br/cadernos/MontaCadPdf1.php?Municipio=85000 >. Acesso: 03 jan. 2019.

KAZDA, J. Occurrence of non-cultivable acid-fast bacilli in the environment and their relationship to $M$. leprae. Leprosy Review., v.52, n.1, p.85-91, 1981. https://doi.org/10.5935/0305-7518.19810061

KAZDA, J.; GANAPATI, R.; REVANKAI, C. Isolation of environment derived Mycobacterium leprae from soil in Bombay. Leprosy Review 1986; 579(3):201-208. https://doi.org/10.5935/03057518.19860111

LACAZ, C. S. Introdução à geografia médica do Brasil. São Paulo: EDUSP, 1972.

LEANO, H. A. M, ARAÚJO K. M. F. A. BUENO I. C. NIITSUMA E. N. A, LANA F. C. F. Socioeconomic factors related to leprosy: an integrative literature review. Rev Bras Enferm. 2019; disponível em< http://dx.doi.org/10.1590/0034-7167-2017-0651> acesso junho de 2020. https://doi.org/10.1590/00347167-2017-0651

MAACK, R. Geografia física do Estado do Paraná. 3.ed. Curitiba: Imprensa Oficial, 2002.

MINEROPAR, Minerais do Paraná. Geologia de planejamento: Caracterização do Meio Físico da Área Urbana de Guarapuava. MINEROPAR: Curitiba, 1992.

MOHANTY, P. S. et al. Viability of Mycobacterium leprae in the environment and its role in leprosy dissemination. Indian J Dermatol Venereol Leprol 2016; 82:23-7 Disponível em: $<$ http://www.ijdvl.com/article.asp?issn=03786323; year=2016; volume=82;issue=1; spage=23; epage=27; aulast=Mohanty\#ref5>. Acesso: 08 de fev. de 2019. https://doi.org/10.4103/0378-6323.168935

MURTO, C; CHAMMARTIN, F; SCHWARZ, K; COSTA, L. M; KAPLAN, C; HEUKELBACH, J. Patterns of migration and risks associated with leprosy among migrants in Maranhao, Brazil. 2013. Disponível em: https://journals.plos.org/ plosntds/article?id=10.1371/journal.pntd.0002422. 30 jun. 2020. https://doi.org/10.1371/journal.pntd.0002422

OLIVEIRA, Ederson Dias et al. Caracterização Morfométrica Da Bacia Hidrográfica Do Rio Cascavel, Guarapuava/PR. Analecta - Guarapuava, 2009. Disponível em: $<$ http://revistas.unicentro.br/index.php/analecta/article/view/2098>. Acesso: 05/02/2019.

PEREHOUSKEI, N. A.; BENADUCE, G. M. C. Geografia da Saúde e as Concepções Sobre o Território. Rev. Gestão \& Regionalidade, 2007.

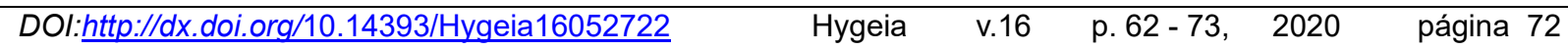


PARANÁ: Secretaria de Saúde: Hanseníase dados preliminares 2019: disponível em<http://www.saude.pr.gov.br/modules/conteudo/conteudo.php?conteudo=3237> Acesso 13 de maio de 2020.

SILVA, J. M. Processos econômico-sociais regionais e seus impactos sobre a estrutura urbana de Guarapuava-PR. Revista de História Regional, UEPG, Ponta Grossa, v.2, n.1, p. 9-42, 1997.

THOMAZ, E. L. VESTENA, L. R. Aspectos Climáticos de Guarapuava-PR. Guarapuava: UNICENTRO, 2003.

WORLD HEALTH ORGANIZATION (WHO). Expert committer on leprosy. Sixth Report. Geneva, 1988. Disponível em: <http://apps.who.int/iris/handle/10665/37409>. Acesso em: 14 mar. 2016.

VESTENA, L. R; SCHMIDT, L. P. Algumas reflexões sobre a urbanização e os problemas socioambientais no centro centro-sul paranaense. Acta Scientiarum, Human and Social Sciences, Maringá, v. 31, n. 1, p. 67-73, 2009. https://doi.org/10.4025/actascihumansoc.v31i1.4589 